\title{
GRAU DE EVIDENCIAÇÃO DO IMPAIRMENT E VALOR DE MERCADO: ANÁLISE DAS EMPRESAS QUE COMPÕEM O ÍNDICE BOVESPA
}

\section{DEGREE OF IMPAIRMENT LOSS DISCLOSURE AND MARKET VALUE: ANALYSIS OF COMPANIES THAT COMPOSE THE BOVESPA INDEX}

\begin{abstract}
0 artigo recebeu o prêmio de melhor trabalho da área de Contabilidade para Usuários Externos, no $2^{\circ}$ Congresso UFU de Contabilidade, realizado nos dias 19 e 20
\end{abstract} de outubro de 2017, na Universidade Federal de Uberlândia

\section{RESUMO}

O presente artigo tem como objetivo verificar se existe relação entre o grau de evidenciação do impairment e o valor de mercado das empresas que compõem o Índice BOVESPA no período entre 2011 e 2015, isto é, isolar o efeito do grau de evidenciação do impairment no valor de mercado. Foram realizadas análises estatísticas quanto ao atendimento às determinações de evidenciação previstas no CPC 01 (R1) e, em seguida, foi realizada uma análise multivariada por meio de regressão envolvendo o Valor de Mercado (VM), como variável dependente, mensurado pelo $Q$ de Tobin, e o grau de evidenciação do impairment. Os resultados da regressão com dados em painel apontam a existência de uma associação positiva entre o grau de evidenciação do impairment e o valor de mercado das empresas analisadas, assim como para as variáveis de controle Oportunidade de Crescimento (OP) e Tamanho da Empresa (TAM). Adicionalmente, identificou-se que o reconhecimento do impairment afeta negativamente o valor de mercado das empresas. Em relação à evidenciação, verificou-se que a média de atendimento aos itens exigidos pelo CPC 01 (R1) aumentou ao longo dos últimos anos e que houve um aumento de $60 \%$ no número de empresas que reconhecem perda por impairment durante o período analisado. Por fim, o aumento do grau de evidenciação do impairment ao longo dos anos pode estar relacionado ao fato de que, quanto maior for esse nível, menor será o impacto negativo do reconhecimento do impairment no valor de mercado das empresas.

Palavras-chave: evidenciação; impairment; valor de mercado.

\begin{abstract}
The purpose of this paper is to verify if there is a relationship between the degree of impairment loss disclosure and the market value of companies listed on the BOVESPA Index, in the period of 2011-2015. Statistical analyses were performed in compliance with the determinations of disclosure set forth in CPC 01 (R1), followed by a multivariate analysis by means of regression involving Market Value (MV) as a dependent variable, measured by Tobin's Q, and the degree of impairment disclosure. Results point to the existence of a positive association between the degree of impairment disclosure and the market value of the firms analyzed, as well as to the correlation between Growth Opportunity (OP) and Firm Size (TAM). In addition, it was possible to support that the recognition of impairment loss negatively affects the market value of the companies. Regarding disclosure, it was verified that the average compliance with the items required by CPC 01 (R1) increased over the last years and that there was a $60 \%$ increase in the number of companies that recognize impairment losses during the analyzed period. Finally, the increase in the degree of impairment disclosure over the years may be related to the fact that the higher this level, the lower the negative impact of the impairment recognition in the market value of companies.
\end{abstract}

Keywords: Disclosure; Impairment loss; Market value.

\section{Térsio Arcúrio Júnior}

Mestre em Ciências Contábeis pela Universidade de Brasília (UnB). Especialista em Auditoria, Controladoria e Perícia Contábil pelo Instituto Brasil Extensão e Pós-Graduação (Ibep) e em Contabilidade Pública e Responsabilidade Fiscal pela Faculdade Internacional de Curitiba (Facinter). Graduado em Ciências Contábeis pela Universidade Paulista (Unip). Especialista em Regulação da Agência Nacional de Telecomunicações (Anatel). Contato: SQSW Qd. 302 Bloco F, Ap. 407, Brasília, DF, CEP 70.673-206. E-mail: tersioarcurio@hotmail.com

\section{Bruno Rodrigues Teixeira de Lima Advogado atuante em contencioso e consultoria fiscal. Bacharel em Direito e especialista em Direito Tributário pela Universidade Católica de Brasília. Bacharel e mestre em Ciências Contábeis pela Universidade de Brasília. Tem experiência em Direito Tributário, Proces- so Tributário e Contabilidade Fiscal. Contato: SQS 415 Bloco E, Ap. 108, Asa Sul, Brasília, DF, CEP: 70.298-050. E-mail:brlima@me.com}

\section{Rodrigo de Souza Gonçalves}

Doutor em Ciências Contábeis pelo Programa Multi-institucional e Inter-regional de Ciências Contábeis UnB/ UFPB/UFRN. Mestre em Controladoria e Contabilidade Estratégica pela Fundação Escola de Comércio Álvares Penteado (Fecap). Professor do Departamento de Ciências Contábeis e do Programa de Pós-Graduação em Ciências Contábeis da Universidade de Brasília (UnB). Contato: Campus Darcy Ribeiro - Prédio da Face - Asa Norte, Brasília, DF, CEP: 70910-900. E-mail: rgoncalves@unb.br

\section{Jomar Miranda Rodrigues}

Doutor e Mestre em Ciências Contábeis (UnB) Especialista em Controladoria e Finanças (UnB). Especialista em Contabilidade Gerencial (Upis). Graduado em Ciências Contábeis (Upis). Docente do curso de Ciências Contábeis e Atuariais da UnB e do Programa de Pós-graduação em Ciências Contábeis (PPGCont-UnB). Contato: Campus Universitário Darcy Ribeiro - Prédio da Face - Sala BT2 47/7 - Asa Norte, Brasilia, DF, CEP: 70910-900. E-mail: jomar@unb.br 


\section{INTRODUÇÃO}

A convergência às normas internacionais tem, entre seus objetivos, o de alcançar maior qualidade da divulgação da informação contábil, haja vista que a divulgação de informações sob um mesmo padrão contábil possibilita uma melhor análise comparativa entre diversas empresas (SANTOS et al., 2011).

Nesse contexto, as leis n. ${ }^{\circ}$ s 11.638/2007 e 11.941/2009 passaram a exigir das empresas brasileiras a adoção de procedimentos aderentes às normas internacionais de contabilidade, contribuindo, portanto, com a convergência às referidas normas. Dentre as práticas contábeis que devem ser adotadas, tem-se o teste de redução ao valor recuperável de ativos, tratado pelo International Accounting Standards Board (IASB), por meio da norma IAS 36 Impairment of Assets, a qual se correlaciona com o Pronunciamento CPC 01 (R1) - Redução ao Valor Recuperável de Ativos.

Cumpre esclarecer que a Redução ao Valor Recuperável de Ativos possui diversas expressões no Brasil, todas derivadas do termo em inglês impairment loss, tais como: perda no valor recuperável, perda por recuperabilidade, perda por imparidade ou perda por desvalorização. Sendo assim, a partir desse ponto, o termo será tratado simplesmente como impairment.

O CPC 01 tem por finalidade assegurar que os ativos estejam reconhecidos por valor que não exceda seus valores de recuperação, caso contrário, há necessidade do reconhecimento do impairment.

Além disso, o CPC 01 (R1) determina a evidenciação das premissas que fundamentam os cálculos do impairment, contribuindo para o alcance da função primordial da contabilidade nos termos de Iudícibus (2010), que é fornecer informações de qualidade aos usuários. O referido autor comenta, ainda, que a evidenciação é um compromisso inalienável da contabilidade com seus usuários e com seus próprios objetivos, já que o cerne da evidenciação é apresentar a informação qualitativa e quantitativa de forma ordenada.

Nesse cenário, nota-se que a importância da evidenciação para as empresas e para o mercado de capitais tem sido objeto de análise de pesquisas anteriores, a exemplo de Sousa et al. (2014), a qual conclui que a evidenciação contábil está associada, de forma significante e positiva, ao valor de mercado das empresas.

Por outro lado, quando se trata de evidenciação de perdas, a relação pode ser contrária, isto é, ao divulgar perdas por impairment, o valor de mercado pode ser afetado negativamente, conforme sugerido nos estudos de Kothari e Barone (2011), Souza, Borba e Zandonai (2011) e de Vogt et al. (2016), nos quais foi encontrada uma relação negativa entre a divulgação do impairment e o valor de mercado da empresa, podendo, ainda, haver a mesma relação negativa com o valor do patrimônio e com as projeções de fluxos de caixa futuros (VOGT et al., 2016).

Vale destacar que, apesar de ser esperado um provável efeito negativo no valor de mercado quando há a divulgação de uma perda possível por impairment, é difícil observar qual seria a relação entre um nível maior ou menor de evidenciação dessa perda e o valor de mercado da empresa. Em outras palavras, observar qual seria o impacto causado sobre o valor de mercado da empresa ao evidenciar com mais completude as informações sobre a perda. Desse modo, apesar dos diversos aspectos já abordados até o momento, ainda há uma lacuna no que diz respeito à análise da relação entre o grau de evidenciação do impairment e o valor de mercado das empresas.

Nesse contexto, como se trata de um tema alinhado ao processo de convergência às normas internacionais e, conforme alertam Souza, Borba e Zandonai (2011), como há uma complexidade na mensuração e no uso do impairment, o presente estudo faz o seguinte questionamento: existe associação entre o grau de evidenciação do impairment e o valor de mercado das empresas?

Vale mencionar que, ao longo dos últimos anos, a relevância do impairment pode ser percebida em decorrência de diversos estudos. Renunciando à exaustividade ao citar alguns aspectos abordados, pode-se encontrar esse tema atrelado: ao gerenciamento de resultado (ALFONS, 2009); a fatores que explicam o nível de evidenciação do impairment (SOUZA, 2011); ao value relevance do impairment do goodwill (XU; ANANDARAJAN; CURATOLA, 2011); a níveis de disclosure (SOUZA; BORBA; ZANDONAI, 2011; ALBANI; ALMEIDA, 2012; MACHADO, 2013); aos conflitos de agência (OLUGBENGA, 2014); à associação entre ativos tangíveis e intangíveis e aos fluxos de caixas futuros (KARAMPINIS; HEVAS, 2014); à divulgação do impairment do goodwill (BARBOSA et al., 2014); aos determinantes do reconhecimento das perdas por impairment do goodwill (VOGT et. al., 2016) e à relação entre a capacidade dos gestores e a deterioração dos ativos (SUN, 2016).

Assim, embora haja diversos estudos sobre a temática impairment, ainda existe uma lacuna na literatura quanto à relação entre o seu grau de evidenciação, quando identificada a perda por impairment, e seus efeitos no valor de mercado das empresas.

Diante disso, este estudo avança em relação aos anteriores ao contribuir para a compreensão da informação divulgada acerca do reconhecimento da perda em relação ao valor de mercado das empresas. Além disso, identifica se as disposições normativas estão sendo cumpridas, o que possibilita aos órgãos reguladores do mercado de capitais a obtenção de subsídios relacionados à atividade de monitoramento do cumprimento das disposições normativas.

Quanto à abordagem metodológica, a presente pesquisa contribui ao identificar os itens evidenciados relativos às exigências do CPC 01 (R1), verificando a aderência ou não aos itens de divulgação do pronunciamento, com a finalidade de construir um indicador de divulgação, bem como ao testar a repercussão dessa divulgação no valor de mercado das organizações. Após isto, foi feita uma análise de regressão multivariada a fim de identificar os determinantes de evidenciação e sua relação com o valor de mercado.

Com isso, o presente artigo está estruturado, logo após esta introdução, com aspectos teóricos, referentes ao impairment e ao valor de mercado das empresas, seguidos pelo percurso metodológico, que se refere ao levantamento e à análise dos dados, e, na sequência, pela análise de resultados e pelas considerações finais sobre a pesquisa. 


\section{REVISÃO DE LITERATURA 2.1 Impairment e Valor de Mercado}

A perda por redução ao valor recuperável, assim disposta no Pronunciamento CPC 01 (R1), é entendida como o montante pelo qual o valor contábil do ativo ou da unidade geradora de caixa excede seu valor recuperável.

Segundo Vogt et al. (2016), o conceito de impairment é tratado sob duas perspectivas. A primeira está relacionada ao valor justo, ou seja, quando o valor justo é inferior ao valor contábil. Nesse caso, se o valor recuperável de um ativo for menor que o seu valor contábil, o valor contábil deverá ser reduzido ao valor recuperável e, portanto, haverá o impairment (MEETING; LUECKE, 2002; SANTOS; MACHADO; SCHMIDT, 2003; SOUZA; BORBA; ZANDONAI, 2011; KOTHARI; BARONE, 2011).

A segunda perspectiva refere-se à perda da capacidade de geração dos benefícios econômicos futuros dos bens, isto é, está associada ao valor em uso do ativo, o qual considera os fluxos de caixa futuros que esse ativo pode gerar durante sua vida útil (SANTOS; MACHADO; SCHMIDT, 2003; SOUZA; BORBA; ZANDONAI, 2011; VOGT et al., 2016). Nesse caso, se o valor dos fluxos de caixa futuro dos bens for inferior ao valor contábil, deve ser reconhecido o impairment.

Dessa forma, o impairment pode ser influenciado pelas variáveis utilizadas para sua mensuração, tais como: fluxos de caixa, estimativa de taxa de crescimento a longo prazo e taxas de desconto de atualização de fluxo de caixa, sendo uma área fértil ao comportamento oportunista (AVALLONE; QUAGLI, 2015), propiciando um cenário de gerenciamento de resultados. Adicionalmente, em decorrência do gerenciamento de resultados, há a possibilidade de prejuízo da qualidade das informações contábeis, pois a utilização do impairment para fins de suavização do resultado, por exemplo, pode influenciar decisões dos usuários da informação, conforme destacado por Amaro et al. (2015), em que um dos objetivos da prática de suavização do resultado é demonstrar uma geração de resultados com menores oscilações, mitigando riscos e incertezas sobre as atividades econômicas.

Vale ressaltar que Barbosa et al. (2014) alertam para o fato de que, se a divulgação for imprecisa e inconsistente, o tomador de decisão será prejudicado. Além disso, asseveram que, devido o cálculo do impairment possuir subjetividade, é importante que as informações sejam fidedignas para permitir a correta avaliação das demonstrações financeiras, assim como revelam os resultados do estudo de Lapointe-Antunes, Cormier e Magnan (2009). Na mencionada pesquisa, verificou-se que há uma relação negativa entre o reconhecimento da perda por impairment e o preço das ações, bem como verificou-se que a noção de representação fidedigna é mais relevante na precificação dos ativos do que a ideia de precisão dos valores reconhecidos.

Esse contexto, aliado ao argumento de Tavares Filho (2006) de que a qualidade das informações divulgadas influencia o valor de mercado de uma empresa, demonstra a importância da qualidade da evidenciação do impairment aos usuários da informação e da necessidade de práticas que representem de forma fidedigna esse evento econômico.

Ainda no que se refere à importância da divulgação da informação contábil, o estudo de Xu, Anandarajan e Curatola (2011) sugere que o impairment do goodwill é geralmente visto como uma informação relevante, porém, à medida que ocorre, o valor de impairment é visto de forma negativa pelo investidor, o que é esperado devido às características que cercam o reconhecimento da referida perda. Desse modo, é esperada uma associação negativa entre a divulgação de impairment e o valor de mercado.

No mesmo sentido, Kothari e Barone (2011), Souza, Borba e Zandonai (2011) e Vogt et al. (2016) sugerem a existência de uma relação negativa entre a divulgação do impairment e o valor de mercado da empresa. No entanto, torna-se importante saber se essa relação também é negativa ao considerar somente o grau de divulgação da perda. Em outras palavras, considerando que houve o reconhecimento de perda por impairment, o questionamento é sobre qual seria a associação entre o grau de evidenciação dessa perda e o valor de mercado. Nesse momento, insere-se a qualidade da informação como um componente que tem por objetivo trazer transparência ao usuário externo quanto ao reconhecimento dos eventos econômicos ocorridos na organização.

Assim, conforme mencionado, é esperado que o valor de mercado seja afetado negativamente pelo reconhecimento da perda por impairment. Entretanto, à medida que há um maior disclosure, isto é, maior grau de evidenciação, este, por sua vez, em razão do papel desempenhado pela informação contábil, que possui maior value relevance, pode vir a ter uma relação positiva com o valor de mercado (BARTH; LANDSMAN; LANG, 2008; KOUKI, 2018), uma vez que contribui, entre outros fatores, para a redução de incertezas e para a acurácia da previsão dos analistas (ANDRÉ; DIONYSIOU; TSALAVOUTAS, 2018).

Normalmente, essa hipótese - relação positiva entre o valor de mercado e a maior divulgação - é mais comum quando se trata de maior disclosure de informações contábeis que contribuem para a compreensão do desempenho econômico corporativo de aspectos não relacionados diretamente às perdas, como é o caso deste estudo. Apesar disso, os achados de André, Dionysiou e Tsalavoutas (2018) revelam, em uma amostra de empresas europeias, que a divulgação dos aspectos relacionados ao IAS 36 (Impairment of Assets) apresentou uma relação positiva com o valor de mercado e negativa com dispersão da previsão dos analistas.

Desse modo, o estudo de André, Dionysiou e Tsalavoutas (2018) corrobora a hipótese de que, ainda que a informação contábil esteja relacionada às perdas, no caso de impairment, considerando que essa possui conteúdo informacional fidedigno, ela contribui para uma maior acurácia na avaliação das empresas e na previsão dos resultados futuros, sustentando-se, portanto, a seguinte hipótese de pesquisa:

H1: Existe associação positiva entre o grau de evidenciação do impairment e o valor de mercado das empresas. 


\section{PERCURSO METODOLÓGICO}

Com a finalidade de identificar as empresas que tiveram perda por impairment reconhecida no resultado, foi efetuado um levantamento, na base de dados Economática ${ }^{\circledR}$, de empresas integrantes que compõem o Índice BOVESPA, referente ao período de 2011 a 2015. Desse cruzamento, resultou o universo de 56 empresas.

A seleção de empresas que compõem o Índice BOVESPA teve por objetivo utilizar informações de empresas que possuem maior negociabilidade e representatividade no mercado acionário brasileiro. Quanto ao período analisado, esse se justifica por representar o início do período seguinte à aprovação do CPC 01 (R1) até o último ano em que as empresas disponibilizavam tais informações.

Considerando as especificidades do estudo, da população de 56 empresas foram excluídas 5 empresas bancárias, devido às particularidades operacionais e contábeis dessas instituições; 1 empresa com dados indisponíveis, e, por fim, foram retiradas 37 empresas que não reconheceram perda por impairment.

Desse modo, para a amostra final, restaram 12 empresas, conforme o Quadro 1.

Quadro 1 - Empresas que reconheceram perda por impairment no período

\begin{tabular}{|c|c|c|c|c|c|}
\hline Empresa & 2011 & 2012 & 2013 & 2014 & 2015 \\
\hline Ambev S/A & $\mathrm{X}$ & & $\mathrm{X}$ & $\mathrm{X}$ & $\mathrm{X}$ \\
\hline Braskem & $\mathrm{X}$ & $\mathrm{X}$ & & & \\
\hline Cesp & & & & $\mathrm{X}$ & \\
\hline Copel & & & & $\mathrm{X}$ & \\
\hline CPFL Energia & & & & & $\mathrm{X}$ \\
\hline Gerdau & & & & $\mathrm{X}$ & $\mathrm{X}$ \\
\hline Petrobrás & $\mathrm{X}$ & $\mathrm{X}$ & $\mathrm{X}$ & $\mathrm{X}$ & $\mathrm{X}$ \\
\hline Qualicorp & & $\mathrm{X}$ & $\mathrm{X}$ & $\mathrm{X}$ & $\mathrm{X}$ \\
\hline Sid Nacional & $\mathrm{X}$ & & $\mathrm{X}$ & & \\
\hline Tractebel & & & $\mathrm{X}$ & & $\mathrm{X}$ \\
\hline Usiminas & $\mathrm{X}$ & $\mathrm{X}$ & & & $\mathrm{X}$ \\
\hline Vale & & $\mathrm{X}$ & $\mathrm{X}$ & $\mathrm{X}$ & $\mathrm{X}$ \\
\hline Total & 5 & 5 & 6 & 7 & 8 \\
\hline
\end{tabular}

O Quadro 1 evidencia que apenas uma empresa reconheceu impairment durante todo o período, além de ser possível notar um aumento na quantidade de empresas que reconheceram impairment ao longo dos anos. Ademais, ressalta-se que, embora o Quadro 1 apresente lacunas quanto ao reconhecimento do impairment, diversas notas explicativas mencionam a realização do referido teste. Portanto, as lacunas indicam que não foram constatadas perdas por impairment.

Para a coleta de dados, foi visitado o site da CVM, no qual constavam as informações financeiras, demonstrações contábeis e notas explicativas. A coleta ainda foi baseada na adaptação do instrumento de pesquisa elaborado por Souza, Borba e Zandonai (2011), conforme o Quadro 2, a seguir.

Quadro 2 - Exigências para a divulgação do impairment, conforme o CPC 01 (R1)

\begin{tabular}{|c|c|c|}
\hline Requisitos & n. ${ }^{\circ}$ & Itens \\
\hline \multirow{3}{*}{ a) Para cada classe de ativos } & 1 & O montante da perda/reversão \\
\hline & 2 & Linha da DRE na qual essas perdas/reversão foram incluídas \\
\hline & 3 & $\begin{array}{l}\text { O montante de perda/reversão por desvalorização de ativos reavaliados } \\
\text { reconhecidos em outros resultados abrangentes }\end{array}$ \\
\hline \multirow{2}{*}{$\begin{array}{l}\text { b) Para entidade que reporta } \\
\text { informação por segmento }\end{array}$} & 4 & $\begin{array}{l}\text { O montante das perdas/reversões por desvalorização reconhecido, durante o } \\
\text { período, na demonstração do resultado }\end{array}$ \\
\hline & 5 & $\begin{array}{l}\text { O montante das perdas/reversões por desvalorização reconhecido, durante o } \\
\text { período, na demonstração do resultado abrangente }\end{array}$ \\
\hline
\end{tabular}




\begin{tabular}{|c|c|c|}
\hline Requisitos & n. ${ }^{\circ}$ & Itens \\
\hline \multirow{13}{*}{$\begin{array}{l}\text { c) Para cada ativo individual, } \\
\text { incluindo ágio por expectativa de } \\
\text { rentabilidade futura, ou unidade } \\
\text { geradora de caixa }\end{array}$} & 6 & $\begin{array}{l}\text { Eventos e circunstâncias que levaram ao reconhecimento ou à reversão da } \\
\text { perda por desvalorização }\end{array}$ \\
\hline & 7 & O montante da perda reconhecida ou revertida \\
\hline & 8 & Para um ativo individual, sua natureza \\
\hline & 9 & $\begin{array}{l}\text { Quando a entidade reportar informações por segmento de acordo com o } \\
\text { CPC 22, o segmento a ser reportado ao qual o ativo pertence }\end{array}$ \\
\hline & 10 & Para uma unidade geradora de caixa, sua descrição \\
\hline & 11 & Para uma unidade geradora de caixa, o montante da perda/reversão \\
\hline & 12 & Para uma unidade geradora de caixa, a informação por segmento \\
\hline & 13 & $\begin{array}{c}\text { Para uma unidade geradora de caixa, a descrição quanto à mudança na } \\
\text { desagregação de ativos, se houver }\end{array}$ \\
\hline & 14 & $\begin{array}{l}\text { Especificar se o valor recuperável do ativo é seu valor justo líquido de despe- } \\
\text { sa de alienação ou seu valor em uso }\end{array}$ \\
\hline & 15 & $\begin{array}{l}\text { Se o valor usado for o valor justo líquido de despesa de alienação, divulgar o } \\
\text { nível de hierarquia do valor justo de acordo com o CPC } 46\end{array}$ \\
\hline & 16 & $\begin{array}{l}\text { Se estiver no nível } 2 \text { ou 3, divulgar a descrição técnica de avaliação usada } \\
\text { para mensurar o valor justo menos as despesas de alienação }\end{array}$ \\
\hline & 17 & $\begin{array}{c}\text { Se estiver no nível } 2 \text { ou 3, cada pressuposto-chave em que a gerência baseou } \\
\text { a sua determinação de valor justo. Divulgar a taxa de desconto se o valor } \\
\text { justo for mensurado pela técnica de valor presente }\end{array}$ \\
\hline & 18 & $\begin{array}{l}\text { Se o valor recuperável for o valor em uso, a taxa de desconto utilizada na } \\
\text { estimativa corrente e na estimativa anterior (se houver) do valor em uso }\end{array}$ \\
\hline \multirow{3}{*}{$\begin{array}{l}\text { d) Unidade geradora de caixa } \\
\text { contendo ágio por expectativa } \\
\text { de rentabilidade futura (goodwill) } \\
\text { ou ativo intangível com vida útil } \\
\text { indefinida }\end{array}$} & 19 & $\begin{array}{l}\text { O valor contábil do ágio por expectativa de rentabilidade futura (goodwill) } \\
\text { alocado à unidade (grupo de unidades) }\end{array}$ \\
\hline & 20 & $\begin{array}{l}\text { O valor contábil dos ativos intangíveis com vida útil indefinida alocado à } \\
\text { unidade (grupo de unidades) }\end{array}$ \\
\hline & 21 & $\begin{array}{l}\text { A base sobre a qual o valor recuperável da unidade (grupo de unidades) tenha } \\
\text { sido determinado (valor em uso ou valor justo líquido de despesas de alienação) }\end{array}$ \\
\hline \multirow{6}{*}{$\begin{array}{l}\text { I - se o valor recuperável da } \\
\text { unidade (grupo de unidades) tiver } \\
\text { sido baseado no valor em uso }\end{array}$} & 22 & $\begin{array}{l}\text { Descrição de cada premissa-chave sobre a qual a administração tenha basea- } \\
\text { do suas projeções de fluxo de caixa para o período coberto }\end{array}$ \\
\hline & 23 & $\begin{array}{l}\text { Descrição da abordagem utilizada pela administração para determinar o } \\
\text { valor sobre o qual estão assentadas as premissas-chave }\end{array}$ \\
\hline & 24 & $\begin{array}{c}\text { Período sobre o qual a administração projetou os fluxos de caixa, baseada em } \\
\text { orçamento ou previsões por ela aprovados }\end{array}$ \\
\hline & 25 & $\begin{array}{l}\text { Quando um período superior a cinco anos for utilizado para uma unidade } \\
\text { geradora de caixa (grupo de unidades), uma explicação do motivo por que } \\
\text { um período mais longo é justificável }\end{array}$ \\
\hline & 26 & $\begin{array}{l}\text { A taxa de crescimento utilizada para extrapolar as projeções de fluxo de } \\
\text { caixa, além do período coberto pelo mais recente orçamento ou previsão }\end{array}$ \\
\hline & 27 & A taxa de desconto aplicada à projeção de fluxo de caixa \\
\hline $\begin{array}{l}\text { II - se o valor recuperável da } \\
\text { unidade (grupo de unidades) } \\
\text { tiver sido baseado no valor justo } \\
\text { líquido de despesas de venda }\end{array}$ & 28 & $\begin{array}{l}\text { As técnicas de avaliação utilizada para determinar o valor justo líquido de } \\
\text { despesas de venda }\end{array}$ \\
\hline
\end{tabular}




\begin{tabular}{|c|c|c|}
\hline Requisitos & n. ${ }^{\circ}$ & Itens \\
\hline \multirow{12}{*}{$\begin{array}{l}\text { III - se o valor justo líquido de } \\
\text { despesas de venda não é determi- } \\
\text { nado, utilizando-se um preço de } \\
\text { mercado observável para unidade } \\
\text { (grupo de unidades) }\end{array}$} & 29 & $\begin{array}{l}\text { Descrição de cada premissa-chave sobre a qual a administração tenha basea- } \\
\text { do a determinação do valor justo líquido de despesas de venda }\end{array}$ \\
\hline & 30 & $\begin{array}{l}\text { Descrição da abordagem utilizada pela administração para determinar o } \\
\text { valor sobre o qual estão assentadas as premissas-chave }\end{array}$ \\
\hline & 31 & $\begin{array}{l}\text { O nível da hierarquia de valor justo no qual a mensuração do valor justo se } \\
\text { classifica em sua totalidade }\end{array}$ \\
\hline & 32 & $\begin{array}{l}\text { Se tiver ocorrido mudança na técnica de avaliação, a mudança havida e as } \\
\text { razões para fazê-la }\end{array}$ \\
\hline & \multicolumn{2}{|c|}{$\begin{array}{l}\text { Se o valor justo líquido das despesas de venda tiver sido determinado, utilizando } \\
\text { projeções de fluxo de caixa descontado, divulgar: }\end{array}$} \\
\hline & 33 & $\begin{array}{c}\text { O período ao longo do qual a administração tenha projetado os fluxos de } \\
\text { caixa }\end{array}$ \\
\hline & 34 & $\begin{array}{l}\text { A taxa de crescimento utilizada para extrapolar as projeções de fluxo de } \\
\text { caixa }\end{array}$ \\
\hline & 35 & A taxa de desconto aplicada às projeções de fluxo de caixa \\
\hline & \multicolumn{2}{|c|}{$\begin{array}{l}\text { Se uma possivel e razoável mudança em uma premissa-chave sobre a qual a admi- } \\
\text { nistração tenha baseado sua determinação de valor recuperável da unidade puder } \\
\text { resultar em valor contábil superior ao seu valor recuperável: }\end{array}$} \\
\hline & 36 & $\begin{array}{l}\text { O montante pelo qual o valor recuperável da unidade (grupo de unidades) } \\
\text { excede seu valor contábil }\end{array}$ \\
\hline & 37 & O valor sobre o qual está assentada a premissa-chave \\
\hline & 38 & $\begin{array}{l}\text { O novo valor sobre o qual deve estar assentada a premissa-chave, após } \\
\text { a incorporação de quaisquer efeitos derivados dessa mudança em outras } \\
\text { variáveis utilizadas para mensurar o valor recuperável, a fim de que o valor } \\
\text { recuperável da unidade fique igual ao seu valor contábil }\end{array}$ \\
\hline \multirow{8}{*}{$\begin{array}{l}\text { e) Divulgação dos valores do } \\
\text { goodwill e outros ativos in- } \\
\text { tangíveis com vida útil indefinida } \\
\text { (AIVI) alocados por múltiplas } \\
\text { unidades geradoras de caixa }\end{array}$} & 39 & O valor contábil do ágio agregado do goodwill alocado a essas unidades \\
\hline & 40 & $\begin{array}{l}\text { O valor contábil agregado dos ativos intangíveis com vida útil indefinida a } \\
\text { essas unidades }\end{array}$ \\
\hline & 41 & Descrição da premissa-chave \\
\hline & 42 & $\begin{array}{c}\text { Descrição da abordagem da administração para determinar o valor sobre o } \\
\text { qual está assentada a premissa-chave }\end{array}$ \\
\hline & \multicolumn{2}{|c|}{$\begin{array}{l}\text { Se uma possível e razoável mudança em uma premissa-chave sobre a qual a admi- } \\
\text { nistração tenha baseado sua determinação de valor recuperável da unidade puder } \\
\text { resultar em valor contábil superior ao seu valor recuperável: }\end{array}$} \\
\hline & 43 & $\begin{array}{l}\text { O montante pelo qual o valor recuperável da unidade (grupo de unidades) } \\
\text { excede seu valor contábil }\end{array}$ \\
\hline & 44 & O valor sobre o qual está assentada a premissa-chave \\
\hline & 45 & $\begin{array}{l}\text { O novo valor sobre o qual deve estar assentada a premissa-chave, após } \\
\text { a incorporação de quaisquer efeitos derivados dessa mudança em outras } \\
\text { variáveis utilizadas para mensurar o valor recuperável, a fim de que o valor } \\
\text { recuperável da unidade fique igual ao seu valor contábil }\end{array}$ \\
\hline
\end{tabular}

Fonte: Adaptado a partir de Souza, Borba e Zandonai, (2011) (inclusões dos itens sublinhados)

As adaptações foram realizadas com o intuito de tornar o instrumento de pesquisa mais completo, de forma a: abranger todos os itens de divulgação exigidos no CPC 01 (R1); facilitar a compreensão das informações obtidas; melhorar o processo de coleta e de tratamento de dados e auxiliar a construção do indicador de evidenciação, conforme disposto na seção 3.2, a seguir.

\subsection{Valor de Mercado (VM)}

Para o cálculo do valor de mercado da empresa, foi utilizado, como proxy, o coeficiente de Tobin utilizado em estudos dessa natureza (BAUER; GUENSTER; OTTEN, 2004; BEINER et al., 2004; LEAL; CARVALHAL DA SILVA, 2005; GOTARDELO, 2006; MELLO, 2007). O valor de mercado é medido pelo coeficiente de Tobin, da seguinte forma:

$$
\mathrm{Q}=\frac{\mathrm{BVA}-\mathrm{BVCE}+\mathrm{MVCE}}{\mathrm{BVA}}
$$


Onde:

$Q$ : é o $\mathrm{Q}$ de Tobin, que representa o valor de mercado da empresa ao final do exercício social;

$B V A$ : é o valor contábil dos ativos ao final do exercício social;

$B V C E$ : é o valor contábil do patrimônio líquido ao final do exercício social; e

MVCE: é o valor de mercado do patrimônio líquido ao final do exercício social.

\subsection{Grau de Evidenciação do Impairment}

Quanto ao grau de evidenciação (GE) das exigências do CPC 01 (R1), foi utilizada metodologia similar à aplicada por Souza (2011), em que foram desenvolvidas métricas a fim de possibilitar a aplicação da numeração 1 para cada item em que a empresa apresentou conformidade com a evidenciação prevista no CPC 01 (R1) e numeração 0 para os itens que não apresentaram conformidade com a evidenciação disposta no CPC 01 (R1).

Em decorrência de os itens exigidos na norma não serem obrigatórios em todos os casos de impairment, esse estudo utilizou 8 métricas de verificação, a fim de estruturar um conjunto de exigências de evidenciação que deveria ser seguido pelas empresas, conforme as características descritas no quadro a seguir:

Quadro 3 - Métricas para mensuração da evidenciação exigida pelo CPC 01 (R1)

\begin{tabular}{|c|c|}
\hline Métricas & Itens analisados, conforme Quadro 2 \\
\hline $\begin{array}{l}\text { A Métrica } 1 \text { é aplicável aos casos de perda do valor recuperável em } \\
\text { Unidades Geradoras de Caixa, mensurada com base no valor justo. }\end{array}$ & $1,2,3,4,6,7,9,10,11,12,14$ e 15 \\
\hline $\begin{array}{l}\text { A Métrica } 2 \text { é aplicável aos casos de perda do valor recuperável em } \\
\text { Unidades Geradoras de Caixa, mensurada com base no valor em uso. }\end{array}$ & $1,2,3,4,7,9,11,12,14$ e 18 \\
\hline $\begin{array}{c}\text { A Métrica } 3 \text { é aplicável ao Ativo Intangível com Vida Útil Indefinida, } \\
\text { mensurado com base no valor em uso. }\end{array}$ & $\begin{array}{c}\begin{array}{c}1,2,3,4,5,6,7,9,10,11,12,14,18,20,21,22,23,2426 \\
\text { e } 27 .\end{array}\end{array}$ \\
\hline $\begin{array}{l}\text { A Métrica } 4 \text { é aplicável ao Ágio por expectativa de rentabilidade futura, } \\
\text { mensurado com base no valor em uso. }\end{array}$ & $\begin{array}{c}1,2,3,4,5,6,7,9,10,11,12,14,18,19,21,22,23,2426 \\
\text { e } 27 .\end{array}$ \\
\hline $\begin{array}{l}\text { A Métrica } 5 \text { é aplicável às Unidades Geradoras de Caixa e ao Ativo In- } \\
\text { tangivel com Vida Útil Indefinida, mensurados com base no valor justo. }\end{array}$ & $1,2,3,4,5,6,7,9,10,11,12,14,15,16,17,18,20,21$ e 28 . \\
\hline $\begin{array}{l}\text { A Métrica } 6 \text { é aplicável às Unidades Geradoras de Caixa e ao Ativo In- } \\
\text { tangivel com Vida Útil Indefinida, mensurados com base no valor em uso. }\end{array}$ & $\begin{array}{c}1,2,3,4,5,6,7,9,10,11,12,14,18,20,21,22,23,24 \\
26 \text { e } 27 .\end{array}$ \\
\hline $\begin{array}{l}\text { A Métrica } 7 \text { é aplicável às Unidades Geradoras de Caixa e ao Ágio por ex- } \\
\text { pectativa de rentabilidade futura, mensurados com base no valor em uso. }\end{array}$ & $1,2,3,4,5,6,7,9,10,11,12,14,18$ e 21 \\
\hline $\begin{array}{l}\text { A Métrica } 8 \text { é aplicável às Unidades Geradoras de Caixa, mensuradas } \\
\text { pelo valor em uso, e ao Ágio por expectativa de rentabilidade futura, } \\
\text { mensurado com base no valor justo. }\end{array}$ & $1,2,3,4,5,6,7,14,19,20$ e 21 \\
\hline
\end{tabular}

Fonte: Dados da pesquisa

Dessa forma, assegura-se que todos os itens presentes no Quadro 2 possuam a mesma importância, uma vez que as métricas de 1 a 7 serão empregadas considerando cada uma das situações em particular. Ao fazer essa discriminação, ao se obter o grau de evidenciação de uma determinada empresa, o percentual obtido de evidenciação diz respeito unicamente aos itens que ela estaria sujeita a evidenciar, e não a todos os itens contidos no Pronunciamento CPC 01 sumarizados por Souza, Borba e Zandonai (2011). Portanto, ao adotar esse procedimento metodológico, esta pesquisa avança em relação ao estudo de Souza, Borba e Zandonai (2011) à medida que as métricas (de 1 a 7) estabelecem o enquadramento ao qual a empresa analisada está submetida e os itens necessários à sua divulgação.

Por exemplo, uma empresa, ao ser enquadrada na Métrica 2, está submetida à obrigatoriedade de evidenciação de um total de 11 itens. Ao evidenciar 5 dos 11 itens referentes ao impairment, o seu respectivo índice de evidenciação será de $45,45 \%(5 / 11 \times 100)$.

Sendo assim, o Grau de Evidenciação (GE) é representado pela seguinte equação:

$$
\text { Grau Evidenciação }{ }_{i t}=\frac{\sum_{i t=1}^{n} d_{i t}}{n}
$$

Onde:

$d_{i t}$ : será 0 para itens do Quadro 2 não cumpridos e 1 para itens do Quadro 2 que forem cumpridos.

n: quantidade total de itens de divulgação aplicáveis à empresa, conforme as métricas estabelecidas no Quadro 3. 


\subsection{Construção do Modelo de Regressão com Dados em Painel}

Para testar a hipótese da pesquisa, um modelo econométrico foi elaborado na forma de painel, conforme a equação a seguir:

$$
\text { VMit }=\beta_{0}+\beta_{1} \text { GExDRECit }+\beta_{2} \text { ENDit }+\beta_{3} \text { OPit }+\beta_{4} \text { TAMit }+\beta_{5} \text { RSAit }+\beta_{6} \text { VOLit }+\beta_{7} \text { DRECit }+\mathrm{u}_{\mathrm{it}}
$$

$V M$ : indica o valor de mercado da empresa i no momento t, mensurado pelo coeficiente de Tobin;

$D R E C_{\mathrm{it}}$ é uma variável Dummy, à qual é atribuído 1 ponto para os anos em que houve o reconhecimento do impairment e 0 , caso contrário; GE $x$ DREC $C_{\mathrm{it}}$ refere-se ao grau de evidenciação da empresa $i$ no momento $t$, que indica o nível de atendimento aos requisitos da norma CPC 01 (R1), multiplicado pela variável DREC ${ }_{\mathrm{it}}$;

$E N D_{\mathrm{itt}}$ : representa o nível de endividamento da empresa $i$ no momento $t$, dado pela relação entre endividamento e ativos totais;

$O P_{\mathrm{it}}$ : indica a oportunidade de crescimento da empresa $i$ no momento $t$, baseada na variação percentual das receitas dos últimos 3 anos;

$T A M_{\mathrm{t}}$ : refere-se ao tamanho da empresa $i$ no momento $t$, mensurado pelo logaritmo natural do valor total dos ativos;

$R S A_{\mathrm{it}}$ : representa a rentabilidade da empresa $i$ no momento $t$, correspondente à razão entre lucro líquido e valor total dos ativos;

$V O L_{\mathrm{it}}$ : indica a volatilidade histórica anualizada do valor das ações da empresa $i$ no momento $t$,

$u_{\mathrm{it}}$ : é o erro aleatório da regressão, sendo $u_{i t} \sim \mathrm{N}\left(0, \sigma^{2}\right)$.

As variáveis de controle (END, OP, TAM, RSA e VOL) foram incluídas no modelo a fim de minimizar possíveis vieses em razão de essas afetarem a variável dependente, conforme estudos anteriores (LEAL; CARVALHAL DA SILVA, 2005; GOMPERS; ISHII; METRICK, 2003; BLACK; JANG; KIM, 2006; MELLO, 2007; KLAPPER; LOVE, 2004; DROBETZ; FIX, 2003; PEROBELLI et al., 2005).

Vale destacar que a variável $G_{i t}$ foi multiplicada pela variável $D R E C_{i j}$, a qual assume 1 ponto para os anos em que determinada empresa reconheceu impairment e 0, caso contrário. Portanto, a variável GExREC $C_{i t}$ tem por objetivo capturar o efeito no valor de mercado do reconhecimento do impairment, considerando a situação de cada empresa para cada ano, isto é, se reconheceu ou não a perda por impairment $\left(D R E C_{i t}\right)$ e em que medida está em conformidade com os itens obrigatórios a serem evidenciados $\left(G E_{i t}\right)$.

Nesse contexto, Yip e Tsang (2007) esclarecem que há duas formas de trabalhar com variáveis dummies multiplicadas por variáveis independentes. A primeira e menos utilizada refere-se à abordagem partilhada, ou seja, multiplicam-se as variáveis independentes pela variável dummy, com o intuito de identificar a participação de cada variável independente na variável dependente.

Já a segunda forma, mais utilizada e usada neste estudo, refere-se à abordagem base. Nesse caso, retira-se uma categoria do modelo, no caso do presente estudo, o $G E_{i p}$ quando não há perda por redução ao valor recuperável e utiliza-se o $G E_{i t}$ somente quando há perda por redução ao valor recuperável.

Assim, como o objetivo deste estudo é analisar o impacto do GE quando há a ocorrência do evento impairment, ou seja, a relação desse evento com o VM, a referida multiplicação se justifica, uma vez que, agindo dessa forma, o coeficiente da variável $\mathrm{GE}_{i t}{ }^{*} D R E C_{i t}$ irá representar somente o efeito do GE quando há perda por impairment reconhecida.

Diante disso, o GE relativo às informações divulgadas quando não há perda por redução ao valor recuperável, como, por exemplo, informação sobre taxa de desconto aplicada às projeções de fluxo de caixa, não será levado em consideração, tendo em vista que esse contexto, de evidenciação de itens do CPC 01 (R1) de empresas que não reconheceram perda por impairment, não faz parte do escopo deste estudo.

Cabe mencionar que a variável $D R E C_{i t}$ foi incluída, de forma isolada, a fim de testar seu impacto no valor de mercado das empresas. Assim, foi atribuído um (1) ponto para os anos em que houve reconhecimento do impairment e nenhum (0) ponto, caso contrário.

Por fim, para a uniformização das informações, todos os dados contábeis foram atualizados para uma mesma data base, por meio do Índice Nacional de Preços ao Consumidor Amplo (IPCA).

\section{ANÁLISE DOS RESULTADOS 4.1 Estatística Descritiva}

Inicialmente, foi elaborada a análise estatística descritiva, a fim de visualizar o comportamento das variáveis do estudo, conforme a seguir. 
Tabela 1 - Estatística descritiva referente aos anos em que houve impairment

\begin{tabular}{cccccccc} 
& $\boldsymbol{V M}$ & TAM & $\boldsymbol{E N D}$ & OP & $\boldsymbol{R S A}$ & VOL & $\begin{array}{c}\text { GEx- } \\
\boldsymbol{D} \boldsymbol{R} \boldsymbol{C} \boldsymbol{C}\end{array}$ \\
\hline Média & 1,5690 & 18,0244 & 0,1613 & 2,1024 & 0,0289 & 0,3915 & 0,5343 \\
Mediana & 1,0041 & 17,9249 & 0,1890 & 2,0872 & 0,0223 & 0,3480 & 0,5500 \\
Desvio-padrão & 1,1190 & 1,6833 & 0,1606 & 0,2409 & 0,0726 & 0,1499 & 0,2232 \\
Mínimo & 0,6168 & 15,0494 & $-0,1264$ & 1,6439 & $-0,1279$ & 0,2047 & 0,1818 \\
Máximo & 4,5099 & 20,6181 & 0,4354 & 2,8005 & 0,1873 & 0,9020 & 0,9333 \\
Observações & 31 & 31 & 31 & $30 *$ & 31 & 31 & 31 \\
\hline
\end{tabular}

Fonte: Dados da pesquisa

* Uma empresa não possuía dados referentes a receitas em dois anos, não sendo possível calcular a oportunidade de crescimento em um período.

Nota-se que a média do GExDREC dos requisitos determinados pelo CPC 01 (R1) é de 53,43\%, isto é, em média, em torno de $53 \%$ das exigências do CPC 01 (R1) são divulgadas. Além disso, a empresa que mais cumpriu as regras ditadas pelo CPC 01 (R1) atendeu cerca de $93 \%$ dos requisitos e a que menos cumpriu atendeu somente $18,18 \%$. Analisando em conjunto o tamanho das empresas, que apresenta um desvio-padrão de 1,68 em torno da média de 18,02, e o grau de evidenciação médio apresentado, verifica-se que, muito embora sejam empresas com porte relativamente semelhante, a prática de evidenciação é ainda um fator que as discrimina, dada a distância entre o mínimo e máximo da variável GExDREC e seu desvio-padrão.

Essa situação observada, alinhada ao que afirmam Barbosa et al. (2014), pode tornar a decisão tomada pelos usuários com base nestas informações imprecisas, haja vista as lacunas observadas pela ausência da informação acerca dos fatores que causaram ou justificaram a perda por impairment.

Por outro lado, empresas com maior nível de divulgação da informação contábil, ao trazerem mais esclarecimentos aos investidores e analistas, podem agregar valor ao conteúdo informacional (maior value relevance) para sua análise e previsões futuras, conforme afirmam Barth, Landsman e Lang (2008) e André, Dionysiou e Tsalavoutas (2018), podendo ser vistas como oportunidades de investimento de menor risco.

Ao considerar o grau de evidenciação por ano, nota-se que, de 2011 a 2015, houve um aumento de $60 \%$ na quantidade de empresas que reconheceram impairment. Além disso, a média de evidenciação dos requisitos exigidos pelo CPC 01 (R1) também tem demonstrado um aumento durante o período analisado. O percentual médio de atendimento das exigências normativas obteve uma média de 35,91\% em 2011 e atingiu 62,36\% em 2015, conforme a Tabela 2 a seguir.

Tabela 2 - Médias anuais de cumprimento dos requisitos de evidenciação das empresas que reconheceram impairment

\begin{tabular}{ccc}
\hline Ano & Qtde de empresas & Média \\
\hline 2011 & 5 & $35,91 \%$ \\
2012 & 5 & $53,45 \%$ \\
2013 & 6 & $57,20 \%$ \\
2014 & 7 & $52,49 \%$ \\
2015 & 8 & $62,36 \%$ \\
\hline
\end{tabular}

Fonte: Dados da pesquisa

Com base na Tabela 2, é possível afirmar que os requerimentos têm sido atendidos com maior rigor, o que proporciona uma melhoria da informação contábil para o usuário, pois informações divulgadas de forma mais completa possibilitam a compreensão do usuário quanto aos motivos das perdas reconhecidas, possibilitando-lhe maior acurácia em suas previsões futuras (ANDRÉ; DIONYSIOU; TSALAVOUTAS, 2018).

\subsection{Análise dos Resultados da Regressão}

Considerando-se as variáveis especificadas no modelo, inicialmente, foi elaborada a matriz de correlação, a fim de identificar possíveis variáveis altamente correlacionadas. Segundo Salvatore e Reagle (2002), quando duas os mais variáveis são altamente correlacionadas, o modelo econométrico pode apresentar problemas de multicolinearidade, tornando complexo o ato de isolar seus efeitos individuais na variável dependente. 
Tabela 3 - Matriz de correlação

\begin{tabular}{ccccccc}
\hline & RSA & VOL & OP & TAM & GExD & END \\
\hline RSA & 1,0 & & & & \\
VOL & 0,6642 & 1,0 & & & & \\
OP & 0,3629 & 0,4195 & 1,0 & & & \\
TAM & 0,1155 & 0,1678 & 0,1523 & 1,0 & & \\
GExD & 0,3921 & 0,2775 & 0,1527 & 0,3204 & 0,0462 & 1,0 \\
END & 0,4519 & 0,3636 & 0,1809 & 0,3003 & 0,03 & \\
\hline
\end{tabular}

Fonte: Dados da pesquisa

Nota-se que as variáveis não possuem altas correlações entre si, sendo que a maior correlação identificada foi entre as variáveis RSA e VOL $(0,664)$. A fim de corroborar os resultados da matriz de correlação, foi calculada a estatística VIF.

Segundo Wooldridge (2012), as estatísticas de Variance Inflation Factor (VIF), cujo valor deve ser inferior a 10, são utilizadas para analisar multicolinearidade em modelos de regressão. Os resultados do VIF para cada uma das variáveis identificaram que não há valores superiores a 5 e, portanto, as variáveis ora especificadas podem ser testadas em conjunto.

A fim de identificar se as variáveis são estacionárias, foram realizados testes de raiz unitária pelos métodos Levin, Lin \& Chut, ADF - Fisher Chi-Square, e PP - Fisher Chi Square. Para as variáveis GE^DREC, END, TAM e VOL não foi possível rejeitar a hipótese de que não havia raiz unitária. Portanto, para tais variáveis, foram realizados novos testes considerando a primeira diferença. Nesse segundo teste, foram rejeitadas as hipóteses de presença de raízes unitárias e, assim, a série é estacionária. Sendo assim, o modelo foi ajustado, ou seja, as variáveis GE^DREC, END, TAM e VOL foram estimadas em primeira diferença.

Quanto à normalidade, o teste de Jarque-Bera apresentou p-valores acima de 0,05, indicando, portanto, uma distribuição normal dos resíduos.

Para a especificação do modelo, foi utilizado o teste de Breusch-Pagan para identificar se ele deve ser estimado na forma pooled, apresentando um p-valor<0,0396. Assim, foi rejeitada a hipótese nula para a estimação no modelo pooled. Ao realizar o teste de Hausman, o resultado apresentou um $p$-valor $<0,05$ e, portanto, rejeita-se a hipótese nula para estimação do modelo com efeitos aleatórios. Portanto, o modelo com efeitos fixos foi usado.

Por fim, em razão dos problemas de heteroscedasticidade e de autocorrelação, o modelo foi estimado pela matriz SUR - Seemingly Unrelated Regressions, a qual gera parâmetros robustos mesmo na presença de autocorrelação e heteroscedasticidade (MEDRADO et al., 2016).

Assim sendo, a seguir são apresentados os resultados da regressão:

Tabela 4 - Resultado da regressão - variável dependente (valor de mercado)

VMit $=\beta_{0}+\beta_{1}$ GExDit $+\beta_{2}$ ENDit $+\beta_{3}$ OPit $+\beta_{4}$ TAMit $+\beta_{5}$ RSAit $+\beta_{6}$ VOLit $+\beta_{7}$ Dit $+\mathrm{u}_{\mathrm{it}}$

\begin{tabular}{|c|c|c|c|c|}
\hline Variáveis & Coeficiente & Erro Padrão & Estatística -t & Prob. \\
\hline Constante & 0,8910 & 0,2972 & 2,9979 & $0,0056^{\star \star \star}$ \\
\hline GExDREC & 0,2296 & 0,1044 & 2,1994 & $0,0363^{\star \star}$ \\
\hline RSA & 0,5016 & 0,8072 & 0,6213 & 0,5394 \\
\hline VOL & $-0,1333$ & 0,1909 & $-0,6987$ & 0,4905 \\
\hline $\mathrm{OP}$ & 0,3436 & 0,1423 & 2,4135 & $0,0226^{\star \star}$ \\
\hline TAM & 1,3613 & 0,6393 & 2,1292 & $0,0422^{\star \star}$ \\
\hline END & $-0,1726$ & 0,6616 & $-0,2609$ & 0,7960 \\
\hline DREC & $-0,3400$ & 0,0893 & $-3,8059$ & $0,0007^{\star \star \star}$ \\
\hline Período Ajustado & 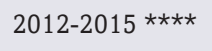 & & & \\
\hline $\mathrm{N}^{\circ}{ }^{\text {de observações }}$ & 47 & & & \\
\hline $\mathrm{R}^{2}$ ajustado & 0,9421 & & & \\
\hline Estatística F & 42,6544 & & & \\
\hline Probabilidade F & 0,0000 & & & \\
\hline
\end{tabular}

Fonte: Dados da pesquisa

Nota: ${ }^{\star \star}, * \star \mathrm{e}^{*}$ denotam a significância estatística das estimativas nos níveis de $1 \%$, 5\% e $10 \%$, respectivamente.

$\star \star \star \star$ Período ajustado devido à correção das raízes unitárias. 
O resultado da regressão demonstra que o $\mathrm{R}^{2}$ Ajustado do modelo explica $94,21 \%$ da variação de VM, enquanto da estatística $\mathrm{F}$ é possível inferir que as variáveis do modelo são conjuntamente significantes para explicar VM, uma vez que a probabilidade ( $\mathrm{p}$-valor) é menor que 0,05 .

Os resultados ainda evidenciam que as variáveis GExDREC, OP, TAM são significativas ao nível de $5 \%$, uma vez que apresentam p-valores de 0,0363, 0,0226 e 0,0422, respectivamente. Dentre as referidas variáveis, é importante destacar a variável Oportunidades de Crescimento (OP), pois, em que pese na amostra existirem empresas que realizam, em algum dos anos analisados (2012-2015), o reconhecimento de perdas por impairment, ainda assim são identificadas empresas com capacidade de crescimento futuro, o que evidencia a relevância da informação contábil, no caso em especial das referidas perdas, a fim de permitir uma melhor acurácia dos analistas/investidores no momento de suas avaliações e previsões futuras (ANDRÉ; DIONYSIOU; TSALAVOUTAS, 2018).

Verifica-se também que, quando há impairment, há um efeito fixo (DREC) e um efeito variável (que depende do GE*DREC), uma vez que as variáveis GExDREC e DREC foram significavas ao nível de $5 \%$ e $1 \%$, respectivamente. Portanto, quando uma empresa apresenta impairment, o efeito fixo sobre o VM apresenta um coeficiente negativo -0,3400 e o efeito variável, que depende do GE da empresa, um coeficiente positivo de 0,02296 para cada 0,1 percentual que a empresa apresente de GE. Assim, no caso de uma eventual empresa possuir o grau de evidenciação igual a 1 (100\%), o efeito total do impairment sobre o VM será de 0,2296 (GExDREC) menos 0,3400 (DREC), isto é, -0,1104.

Nesse contexto, nota-se que, quanto maior for a evidenciação do impairment, isto é, a divulgação dos fatores relacionados ao reconhecimento da perda aos usuários da informação, menor será o impacto negativo sobre o VM decorrente do reconhecimento do impairment.

Esse resultado é relevante não somente no que tange ao fato de se identificar o valor da informação a respeito dos procedimentos adotados para o reconhecimento do impairment em relação ao valor de mercado, mas também pelo fato de que, considerando-se as empresas da amostra, verifica-se que tal efeito ocorre independentemente de a empresa estar inserida em setores considerados de maior regulação no Brasil. Adicionalmente, ao considerar o nível de divulgação apresentado pelas empresas da amostra (média de 53\%), há pelo menos 2 aspectos a serem considerados: a) na medida em que a informação contribui para o esclarecimento dos procedimentos adotados no reconhecimento de impairment, ainda que não divulgados em sua totalidade, ela é relevante, e b) como consequência do item anterior, as empresas deveriam ser estimuladas, por intermédio dos auditores independentes e/ou mecanismos regulatórios, a aumentar o grau de evidenciação quando do reconhecimento das perdas por impairment, uma vez que se trata de uma informação de valor (BARBOSA et al, 2014; KOUKI, 2018).

Portanto, considerando a amostra e o período analisado, uma maior evidenciação do impairment atenua o efeito negativo do reconhecimento da perda. Tal fato, portanto, não rejeita a H1 deste estudo de que existe uma associação positiva entre o GE o VM.

Esses resultados vão ao encontro do argumento de Tavares Filho (2006) de que as informações divulgadas influenciam o valor de mercado de uma empresa, tornando importante a qualidade da evidenciação do impairment aos usuários da informação contábil.

Em relação ao resultado relacionado à DREC, foi apurada uma associação negativa entre o reconhecimento do impairment e o valor de mercado, de outro modo, para cada 1 unidade monetária de reconhecimento do impairment, há um impacto negativo no valor de mercado de -0,3400 unidade monetária, conforme o coeficiente estimado (DREC, tabela 6), corroborando o estudo de Xu, Anandarajan e Curatola (2011), haja vista que valores do impairment são vistos negativamente pelos investidores, o que pode ocasionar uma associação negativa entre o reconhecimento da perda e o valor de mercado. Esse resultado, especialmente o sinal apresentado, corrobora os procedimentos metodológicos adotados na pesquisa, não só em relação aos critérios estabelecidos para a construção da variável de evidenciação (GE), mas, sobretudo, pela variável GExDREC, cujo propósito foi conhecer somente o efeito do grau de evidenciação quando há perda por impairment reconhecida, e não o seu valor ou a ocorrência do evento que foi medido pela variável DREC.

No que tange às demais variáveis, a associação positiva entre OP e VM corrobora as afirmações de Klapper e Love (2004), Beiner et al., (2004) e Black, Jang e Kim (2006), de que há uma relação positiva entre a OP e o VM. Além disso, corrobora os resultados encontrados por Mello (2007).

No que se refere à variável TAM, também se nota a existência de uma relação positiva com o valor de mercado das empresas, uma vez que, para cada aumento de 1 unidade monetária na variável TAM, haverá uma variação positiva no valor de mercado de 1,3613 (variável TAM, tabela 6). Esse resultado vai ao encontro dos estudos de Gompers, Ishii e Metrick (2003) e Leal e Carvalhal da Silva (2005), os quais constatam uma associação positiva entre TAM e VM.

Por fim, em decorrência de os $p$-valores das variáveis END, RSA e VOL não serem significativos $(p>0,05)$ para explicar a variação de VM, não há relação relevante entre tais variáveis e o valor de mercado. Esses achados são compatíveis com os estudos de Drobetz e Fix (2003) e opostos aos estudos de Black, Jang e Kim (2006), Leal e Carvalhal da Silva (2005) e Mello (2005).

\section{CONCLUSÕES}

Com o intuito de testar se há associação entre o GE e o VM das empresas, este estudo utilizou um modelo de regressão linear múltipla, com dados em painel, de forma a possibilitar a identificação do grau de cumprimento dos 
requisitos de evidenciação do impairment exigidos pelo CPC 01 (R1), assim como avaliar se há associação entre o GE e o VM e qual o seu tipo.

Diante disso, foram incluídas as variáveis independentes: END, RSA, OP, TAM, e VOL e, por fim, uma variável dummy, a fim de identificar a associação entre o reconhecimento do impairment e o VM.

Como resultado, a hipótese do estudo - se existe associação positiva entre o GE e o VM das empresas que compõem o Índice BOVESPA - não pôde ser rejeitada. Além disso, foram identificadas associações positivas e significantes da variável VM com as variáveis TAM e OP.

Por outro lado, as demais associações, isto é, os testes de associação entre a variável VM com as variáveis RSA, END e VOL não foram estatisticamente significantes e, portanto, reporta-se que não há relação relevante.

Em relação à variável DREC, foi possível identificar uma associação negativa com a variável VM. Nos períodos em que há reconhecimento do impairment, o valor de mercado é afetado negativamente.

Cumpre ressaltar que, por meio da estatística descritiva, foi possível identificar que a média de cumprimento dos itens exigidos pelo CPC 01 (R1) quanto à divulgação de informações relacionadas à perda por redução ao valor recuperável tem aumentado ao longo dos anos, assim como o número de empresas que reconhecem a perda. Em 2011 , por exemplo, considerando a amostra deste estudo, somente 5 empresas reconheceram a perda e tiveram como média $35,91 \%$ de cumprimento das exigências da norma. De 2012 a 2015, esses percentuais foram de 53,45\%, 57,20\%, 52,49\% e $62,36 \%$ respectivamente, e o número de empresas chegou a 8 em 2015, demonstrando uma tendência de aumento da divulgação a respeito do reconhecimento do teste de impairment, que, por sua vez, foi relevante na análise dos usuários externos ao estar positivamente relacionada com o valor de mercado das empresas.

Diante desse contexto, é possível inferir que o aumento do nível de evidenciação do impairment ao longo dos anos pode estar relacionado ao fato de que, quanto maior for esse nível, menor será o impacto negativo do reconhecimento do impairment no valor de mercado das empresas.

Quanto às contribuições desta pesquisa para a literatura contábil, pode-se destacar: (1) melhor compreensão acerca do resultado das decisões empresariais quanto ao nível de evidenciação das perdas por impairment no valor de mercado; (2) identificação do nível em que as disposições do Pronunciamento CPC 01 (R1) estão sendo cumpridas no âmbito das empresas listadas no IBOVESPA; (3) evidenciação da relevância de uma maior divulgação acerca do reconhecimento das perdas por impairment.

Dessa forma, os resultados deste estudo constituem um mecanismo de argumento para os reguladores do mercado de capitais e de auditorias independentes quanto à necessidade de um melhor cumprimento do Pronunciamento CPC 01 (R1).

Destaca-se, ainda, que a presente pesquisa encontra algumas limitações no que se refere à escolha das variáveis, uma vez que existem outros modelos que buscam analisar o impacto de variáveis no valor de mercado, além do reduzido número de observações, uma vez que a população compreende 56 empresas integrantes do índice IBOVESPA, mas somente 12 apresentaram impairment, equivalendo a, aproximadamente, $22 \%$ do universo da pesquisa.

Nesse sentido, trabalhos futuros podem: (1) adicionar outros modelos que capturem o efeito do nível de evidenciação do impairment no valor de mercado; (2) comparar o mercado brasileiro com outros mercados e (3) incorporar variáveis que possam explicar o comportamento dos gestores, já que isso interfere na decisão de divulgar ou não uma perda.

\section{REFERÊNCIAS}

ALBANI, P. C.; ALMEIDA, J. E. F. de. Teste de impairment de ativos: análise comparativa da evidenciação das companhias abertas listadas no Novo Mercado por setor da economia e auditorias. In: CONGRESSOP USP DE INICIAÇÃO CIENTÍFICA EM CONTABILIDADE, 9., 2012. São Paulo. Anais... São Paulo: USP, 2012. p. 1-14. Disponível em: <http://www.congressousp.fipecafi.org/anais/ artigos122012/598.pdf.> Acesso em: 10 mai. 2016.

ALFONS, M. H. Goodwill Impairment and Earnings management: Do firms use goodwill impairments in big bath accounting? 2009. $32 \mathrm{f}$. Dissertação (MSc in Accountancy \& Control Accountancy Track) - Faculty of Economics and Business University of Amsterdam, Amsterdã, 2009

AMARO, H. D. et al. Impairment e alisamento de resultados em companhias abertas do setor de energia elétrica do Brasil. Revista Mineira de Contabilidade - RMC, v. 16, n. 1, p. 18-26, jan./abr. 2015.

ANDRÉ, P.; DIONYSIOU, D.; TSALAVOUTAS, I. Mandated disclosures under IAS 36 Impairment of Assets and IAS 38 Intangible Assets: value relevance and impact on analysts' forecasts. Applied Economics, v. 50, n. 7, p. 707-725, 2018.

AVALLONE, F. QUAGLI, A. Insight into the variables used to manage the goodwill impairment test under IAS 36. Advances in Accounting, incorporating Advances in International Accounting, v. 31, p. 107-114, 2015.

BARBOSA, J. da S. et al. Impairment no Goodwill: uma análise baseada na divulgação contábil. Revista de Educação e Pesquisa em Contabilidade - REPeC, v. 8, n. 2, p. 159-179. abr./jun. 2014.

BARTH, M. E.; LANDSMAN, W. R.; LANG, M. H. International accounting standards and accounting quality. Journal of accounting research, v. 46, n. 3, p. 467-498, 2008.

BAUER, R.; GUENSTER, N.; OTTEN, R. Empirical evidence on corporate governance in Europe: The effect on stock returns, firm value and performance. Journal of Asset management, v. 5, n. 2, p. 1-104, 2004. 
BEINER, $\mathrm{R}$ et al. An integrated framework of corporate governance and firm valuation - evidence from Switzerland. ECGI - Finance Working Paper, n. 34, p. 1-38, 2004.

BLACK, B; JANG, H; KIM, W. Does corporate governance predict firms' market values? Evidence from Korea. Journal of Law, Economics \& Organization, n. 22, p. 336-413. 2006.

BRASIL. Lei $n .{ }^{\circ} 11.638$, de 28 de dezembro de 2007. Altera e revoga dispositivos da Lei n.․ 6.404, de 15 de dezembro de 1976, e da Lei n.․ 6.385, de 7 de dezembro de 1976, e estende às sociedades de grande porte disposições relativas à elaboração e divulgação de demonstrações financeiras. Disponível em: http://www.planalto.gov.br/ccivil_03/_ato2007-2010/2007/lei/l11638.htm. Acesso em: 21 de abril de 2016

BRASIL. Lei $n .^{\circ} 11.941$, de 27 de maio de 2009. Altera a legislação tributária federal relativa ao parcelamento ordinário de débitos tributários; e dá outras providências. Disponível em: <http://www.planalto.gov.br/ccivil_03/_ato2007-2010/2007/lei/l11638.htm>. Acesso em: 26 de abril de 2016.

COMITÊ DE PRONUNCIAMENTOS CONTÁBEIS (CPC). Pronunciamento técnico CPC 01 (R1) - Redução ao Valor Recuperável de Ativos. Brasília, 2010. Disponível em: <http://static.cpc.mediagroup.com.br/Documentos/27_CPC_01_r1_rev\%2008.pdf>. Acesso em: 20 de abril de 2016.

DROBETZ, W.; FIX, R. What are the determinants of the capital structutre? Some evidence for Switzerland. WWZ/Departament of Finance, Working Paper n. ${ }^{\circ}$ 4/03. University of Basel, 2003. Disponível em: < https://www.semanticscholar.org/paper/What-are-the-Determinants-of-the-Capital-Structure\%3F-Drobetz-Fix/dc567b3e6241fd74705c740fbc77ee951953263e> Acesso em: 10 de abril de 2016 .

GOMPERS, P. A.; ISHII, J. L.; METRICK, A. Corporate Governance and Equity Prices. Quarterly Journal of Economics.v. 118. n. 1. p. 107-155. 2003.

GOTARDELO, D. R. Estudo das práticas de governança corporativa e o desempenho organizacional: uma análise envolvendo rentabilidade, volatilidade e valor de mercado. 2006. 142 f. Dissertação (Mestrado em Administração) - Faculdade de Administração da PUC, Belo Horizonte, 2006.

INTERNATIONAL ACCOUNTING STANDARD BOARD. IAS 36 - Impairment of Assets. Disponível em: < http://eifrs.ifrs.org/ eifrs/bnstandards/en/2016/ias36.pdf> Acesso em: 10 de abril de 2016.

IUDÍCIBUS, S. Teoria da Contabilidade. 10 ed. São Paulo: Editora Atlas, 2010.

KARAMPINIS, N. I.; HEVAS, D. L. Effects of the asymmetric accounting treatment of tangible and intangible impairments in IAS36: International evidence. The Journal of Economic Asymmetries, v. 11, p. 96-103, 2014.

KLAPPER, L. F; LOVE, I. Corporate Governance, Investor Protection, and Performance in Emerging markets. Journal of Corporate Finance. v. 10, n. 5, p. 703-728, 2004.

KOTHARI, J.; BARONE, E. Advanced Financial Accounting: An International Approach Paperback. Financial Times/ Prentice Hall, 2011.

KOUKI, A. IFRS and value relevance: a comparison approach before and after IFRS conversion in the European countries. Journal of Applied Accounting Research, v. 19, p. 60-80, 2018.

LAPOINTE-ANTUNES, P.; CORMIER, D.; MAGNAN, M. Value relevance and timeliness of transitional goodwill-impairment losses: Evidence from Canada. The International Journal of Accounting, v. 44, n. 1, p. 56-78, 2009.

LEAL, R; CARVALHAL DA SILVA, A. Corporate Governance and Value in Brazil (and in Chile). Inter-American Development Bank. Research Network Working paper R-514. Washington, DC, United States, 2005. Recuperado em: <https://papers.ssrn.com/sol3/papers. cfm?abstract_id=726261.> Acesso em: 15 de abril de 2016 .

MACHADO, E. A. et al. Evidências de Disclosure de Valor Recuperável de Ativos em Firmas Listadas no Mercado Acionário Brasileiro. Revista Universo Contábil, v. 9, n. 1, p. 86-103, jan./mar. 2013.

MEDRADO, F. et al. Relação entre o nível de intangibilidade dos ativos e o valor de mercado das empresas. Revista de Contabilidade e Organizações, vol. 10, n. 28, p. 32-44, 2016.

MEETING, D. T.; LUECKE, R. W. Asset impairment and Disposal. Financial Reporting. Journal of Accountancy. v. 193, n. 3, p- 4960, mar. 2002

MELLO, J. S. F. de. O impacto da governança corporativa no valor de Mercado das companhias de capital aberto no Brasil: uma reaplicação. 2007. 107 f. Dissertação (Mestrado em Administração) - Universidade Federal de Minas Gerais, 2007.

OLUGBENGA, O. et al. Financial reporting and compliance of impairment of noncurrent Assets in the nigerian banks. European Journal of Accounting Auditing and Finance Research, v. 2, n. 2, p.18-35, 2014.

PEROBELLI, F. et al. Investigação dos Fatores Determinantes da Estrutura de Capital e da Governança corporativa: Um Enfoque Abordando a Questão da Endogeneidade. In: ENCONTRO NACIOANL DA ANPAD (ENANPAD), 29. 2005. Anais... Brasília: ANPAD, 2005. p. 1-16. Disponível em: <http://www.anpad.org.br/admin/pdf/enanpad2005-ficd-2422.pdf>. Acesso em: 12 mai. 2016. 
SALVATORE, D.; REAGLE, D. Statistics and econometrics. Schaum's Outline Series. New York: ed. The McGraw-Hill Companies, Inc. 2002.

SANTOS, J. L. dos; MACHADO, N. P.; SCHMIDT, P. Teste de impairment para ativos de longa duração: tratamento contábil de acordo com o SFAS n ${ }^{\circ}$ 144. Revista ConTexto. Porto Alegre, v. 3, n. 5, 2 sem. 2003.

SANTOS, P. G. dos et al. Efeito da Lei 11.638/07 sobre o conservadorismo condicional das empresas listadas BM\&FBOVESPA. Revista de Contabilidade e Finanças, v. 22, n. 56, p. 174-188, maio-ago. 2011.

SOUSA, C. B. et al. Valor de Mercado e Disclosure Voluntário: estudo empírico em companhias listadas na BM\&FBOVESPA. Revista Ambiente Contábil, v. 6, n. 2, p. 94-115, jul./dez. 2014.

SOUZA, M. M. de; BORBA, J. A.; ZANDONAI, F. Evidenciação da Perda no Valor Recuperável de Ativos nas Demonstrações Contábeis: uma Verificação nas Empresas de Capital Aberto Brasileiras. Revista Contabilidade Vista \& Revista, v. 22, n. 2, p. 67-91, abr./ jun. 2011.

SOUZA, Maíra Melo de. Perda no Valor Recuperável de Ativos: fatores explicativos do nível de evidenciação das empresas de capital aberto brasileiras. 2011. 127 f. Dissertação de mestrado (Mestrado em Ciências Contábeis) - Universidade Federal de Santa Catarina, 2011.

SUN, L. Managerial ability and goodwill impairment. Advances in Accounting, incorporating Advances in International Accounting, v. 32, p. 42-51, 2016.

TAVARES FILHO, Francisco. Rentabilidade e valor das companhias no Brasil: uma análise comparativa das empresas que aderiram aos níveis de governança corporativa da Bovespa. 2006. 160 f. Dissertação de mestrado (Mestrado em Ciências Contábeis) - Universidade de São Paulo, 2006.

VOGT, M et al. Determinantes do Reconhecimento das Perdas por Impairment do Goodwill. Revista de Contabilidade e Finanças, v. 27, n. 72, p. 349-362, set./dez. 2016.

WOOLDRIDGE, J. M. Introductory Econometrics. A modern Approach. 5th edition. Mason: ed. South-Western/CENGAGE Learnng. 2012.

XU, W.; ANANDARAJAN, A. CURATOLA, A. The value relevance of goodwill impairment. Research in Accounting Regulation, v. 23, p. 145-148, 2011.

YIP, P. S. L.; TSANG, E. W. K. Interpreting dummy variables and their interaction effects in strategy research. Strategic Organization, v. 5, n. 1, p. 13-30, 2007. 